\title{
THE CHARACTERISTICS OF TURBULENT SURFACE FLOW IN PLANAR CHANNEL WITH IMPOSED WALL BLOWING
}

\author{
Y. Na and C. Lee \\ Konkuk University \\ Gwangjin-gu, Hwayang-dong 1, Seoul 143-701, Korea
}

A recent study for cold flow development in an idealized hybrid rocket motor showed that energy spectra of streamwise velocity in the vicinity of the wall exhibited a peculiar local peak at a certain frequency. It is believed that the occurrence of this particular frequency, or time scale, is a direct consequence of flow modification resulting from the interaction of the main oxidizer flow with wall blowing during the process of regression. If external disturbances resulting from white noise or modified injector geometry are imposed on this hybrid system, a sudden amplification of flow instability may be realized in the form of violent oscillation. In this study, a Large Eddy Simulation (LES) technique was used to investigate the response of the flow in the vicinity of the propellant surface to the external forcing applied to the flow field. Results showed that both concentrated and distributed momentum forcings used in the present study did not lead to a sudden growth of instability or disastrous oscillation. However, the pressure field is thought to be more sensitive to the external forcing and exhibits relatively larger oscillations.

\section{NOMENCLATURE}

$C_{S} \quad$ model coefficient for velocity field

$C_{T}$ model coefficient for passive scalar

$E \quad$ energy

$h$ half-channel height

$f$ frequency

$L_{x} \quad$ domain size in the streamwise direction

$L_{y} \quad$ domain size in the wall-normal direction, $2 h$

$L_{z} \quad$ domain size in the spanwise direction

$p$ pressure

Pr molecular Prandtl number

This is an Open Access article distributed under the terms of the Creative Commons Attribution-Noncommercial License 3.0, which permits unrestricted use, distribution, and reproduction in any noncommercial medium, provided the original work is properly cited. 


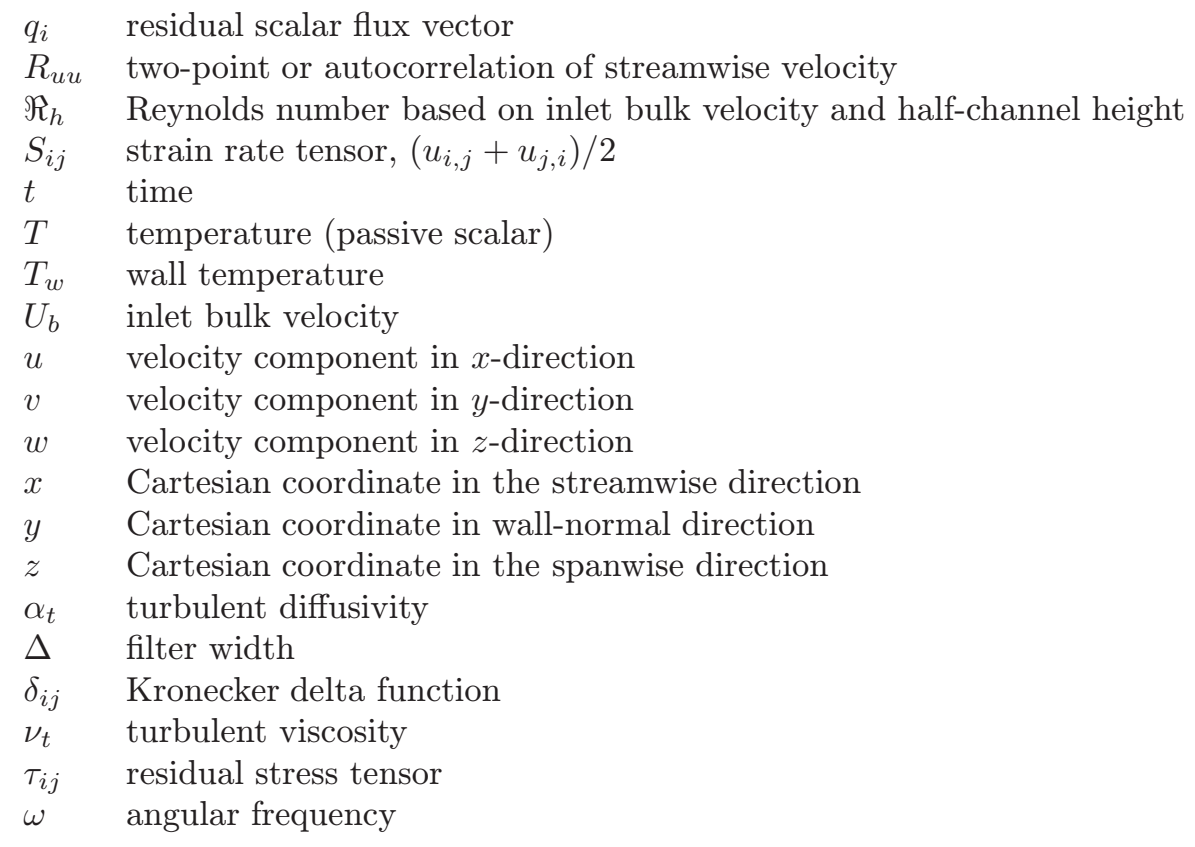

\section{Subscripts}

$b \quad$ bulk mean quantity

mean averaged quantity over time and spanwise direction

rms root mean square quantity

\section{Superscripts}

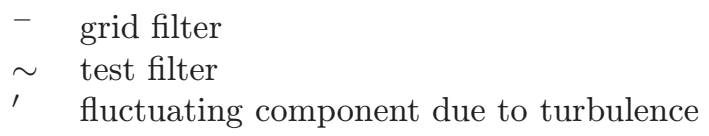

\section{INTRODUCTION}

These days, hybrid rockets attract attention to their excellent safety, relatively high performance, and low development cost. However, their lower density specific impulses as compared to solid rocket motors need to be improved in order to make the propulsion system efficient. Much effort is currently being made towards improving the regression rates of hybrid fuels.

Recent studies with LES methodology [1-3] showed very interesting features of flow behavior near the wall surface. The calculation results indicated that the 
interaction of oxidizer turbulent flow with the wall injected flow can completely alter the structure of turbulent boundary layer and produce isolated cell-like contours of streamwise velocity near the surface. The footprints of streamwise vortices on the surface showed quantitatively the same transient patterns as dark spots observed in the combustion tests $[4,5]$.

From the hydrodynamic point of view, the dynamics of the flow in a solid propellant rocket is believed to be similar to that in hybrid rocket as the mean axial flow and the flow injected from the surface interact with each other in the combustion chamber. French research groups concentrated extensively on the flow instability appearing in solid propellant motors [6]. Also, extensive numerical investigations based on LES of nonreacting flows had been performed in [7-9]. The emphasis was put on the transition and hydrodynamic instability of the flow, and very useful information was reported on turbulence development and acoustic excitation processes in a solid rocket motor with one closed end. Apte and Yang [7-9] claimed that the flow was essentially two-dimensional (2D) in the upstream region of solid rocket motor with the dominant frequencies of vortex shedding governed by hydrodynamic instability waves. In the absence of the appreciable convection effect due to the high-speed stream of oxidizer in their study, however, its flow dynamics was not likely to be the same as in the present situation of the present study where the main oxidizer flow is introduced into the chamber at a relatively high speed and then interacts with the surface injection.

Even though no research has been reported on this subject, it is not surprising to expect that the hydrodynamic instabilities could be possibly developed in the hybrid rocket due to the interaction between the mean and injecting flows. Table 1 summarizes the similarities and differences of flow dynamics in the combustion chamber between solid and hybrid rockets.

In spite of the basic difference in flow configurations between two different rocket motors, the presence of vortex shedding is their common feature. As expected, vortex shedding with a specific frequency occurs due to the presence of shear layer which forms due to the interaction of the main oxidizer flow with the wall blowing in the course of hybrid rocket combustion. Several earlier LES results for solid rockets showed that the onset of hydrodynamic instability could significantly change the unsteady flow evolution if the external disturbances were

Table 1 Summary of flow characteristics in solid and hybrid rocket chambers

\begin{tabular}{lcc}
\hline \multicolumn{1}{c}{ Characteristics } & Solid rocket & Hybrid rocket \\
\hline Axial flow & Injecting driven flow & Fully turbulent oxidizer flow \\
Flow regime & Laminar, transition, turbulent & Turbulent flow \\
Flow oscillation & With a certain frequency & $?$ \\
Response to excitations & Amplification & $?$ \\
\hline
\end{tabular}


superimposed on the flow field. Thus, it is quite interesting to check what would happen if external perturbations are coupled with hydrodynamic instability in the hybrid rocket.

Motivated by this question related to flow instability in hybrid rocket combustion, the LES study has been conducted to find out whether hydrodynamic instability grows rapidly similarly to frequent observations in solid rocket combustion. The main objective of the present work was to study the effect of the external disturbance on the evolution of turbulent flow. Thus, external disturbances in the form of momentum forcing were imposed on the turbulent inflow. Special attention was paid to the response of both streamwise velocity and pressure to these disturbances to identify possible amplification phenomena in the vicinity of the surface.

\section{NUMERICAL METHODOLOGY}

For numerical convenience, a model rocket motor was idealized to be a simple channel and the regression process was approximated by the artificial injection of the fluid through the motor surface as in [3].

\subsection{Computational Geometry and Governing Equation for Large Eddy Simulation}

The geometry of the present injection-driven flow contained several regions with nonnegligible gradients in the wall-normal direction, which required careful resolution. The Reynolds number based on the inlet bulk velocity of the oxidizer and half-channel height was set to 22,500 , which was very close to that of the accompanying experiment [5]. The authors' earlier results indicated that calculations with a higher resolution beyond $1025 \times 193 \times 513$ grid size did not change the overall flow characteristics and thus it was assumed that the mesh size with $1025 \times 193 \times 513$ grids (about 101 million grids) was good enough for the purpose of the present work. The details of numerical calculations and computational grid can be found in $[3,10]$.

Figure 1 shows a schematic of the three-dimensional (3D) computational domain. The streamwise and spanwise dimensions of the domain are $L_{x}=26 h$ and $L_{z}=6.5 h$, respectively, where $h$ is the half-channel height. To provide physically realistic turbulence in the region of interest with linear wall injection, a periodic simple channel (without wall injection) with a length of about $12.2 \mathrm{~h}$ (equivalent to 1800 wall units) was placed ahead of the region with the injectiondriven flow regime. The purpose of this recycled channel was to continuously generate the unsteady turbulence. Following the popular methodology of Lund 


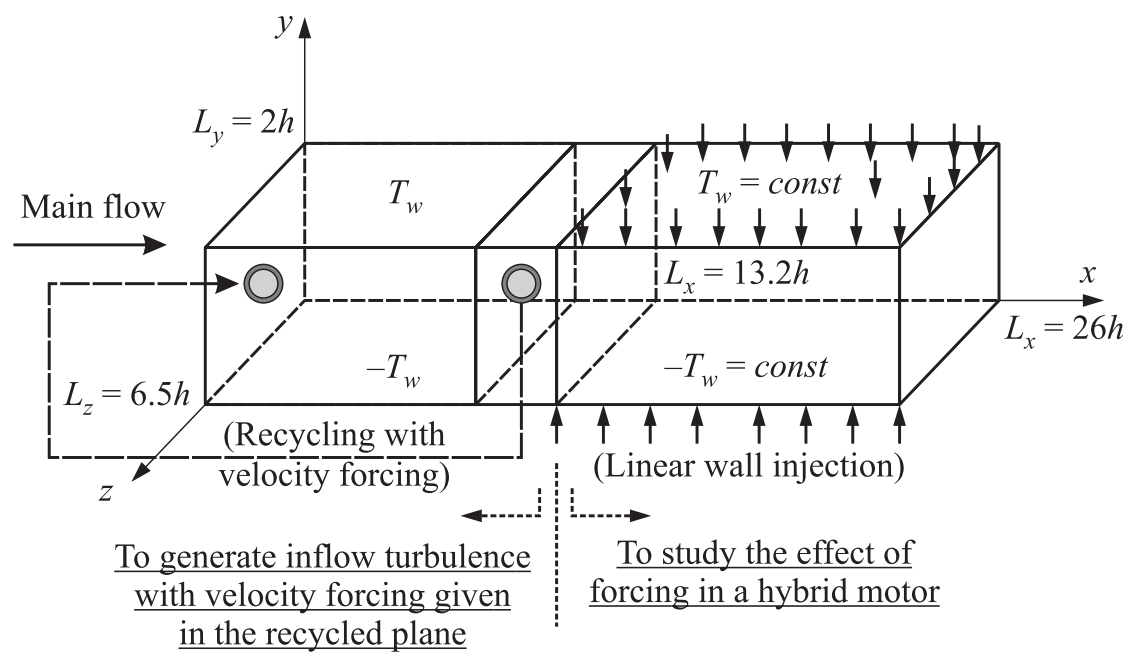

Figure 1 Schematic of computational domain

et al. [11] and the authors' work [10], a streamwise length of about $12.2 \mathrm{~h}$ is good enough for capturing physically realistic turbulent structures which are typically observed in turbulent channel flows.

Assuming that the Mach number in the rocket motor was less than 0.1 , the following set of nondimensional governing equations for velocity and temperature were solved on a rectangular staggered grid:

$$
\begin{aligned}
& \frac{\partial \bar{u}_{i}}{\partial x_{i}}=0 \\
& \frac{\partial \bar{u}_{i}}{\partial t}+\frac{\partial}{\partial x_{j}}\left(\bar{u}_{i} \bar{u}_{j}\right)=-\frac{\partial \bar{p}}{\partial x_{i}}+\frac{\partial}{\partial x_{j}}\left(2 \nu \overline{S_{i j}}-\tau_{i j}\right) \\
& \frac{\partial \bar{T}}{\partial t}+\frac{\partial}{\partial x_{j}}\left(\bar{u}_{j} \bar{T}\right)=\frac{\partial}{\partial x_{j}}\left(\alpha \frac{\partial \bar{T}}{\partial x_{j}}-q_{j}\right) .
\end{aligned}
$$

All the variables were made dimensionless using a bulk velocity at inlet $\left(U_{b}\right)$, upper-wall temperature $(T)$, and half-channel height $(h)$. The fluid density was assumed to be independent of temperature so that the heat was essentially transported as a passive scalar without producing the buoyant force. The details of the numerical procedures of getting the solution and LES algorithms using a dynamic mixed model (DMM) are described in $[3,10]$.

No-slip boundary condition was assumed along the wall except in the second half of the channel (i.e., at $x / h<13$ ) where wall injection was applied. The magnitude of the injection velocity was prescribed to vary linearly from $1 \%$ (at $x / h=13.2$ ) to $3 \%$ (at $x / h=26$ ) of the bulk velocity of the oxidizer at the 
inlet of the computational domain. In the combustion test with poly(methyl methacrylate) (PMMA) fuel conducted by Koo and Lee [5], the blowing velocity (calculated using the data on the average regression rate) was estimated to be about $3 \%$ of the entering oxidizer velocity. Therefore, the blowing velocity was assumed to vary gradually from $1 \%$ to $3 \%$ of the inlet mean flow to minimize the numerical problems associated with the discontinuity in the blowing velocity at $x / h=13.2$. For the calculation of the passive scalar, constant temperatures were maintained along the walls with the lower wall at $T$ and the upper wall at $-T$. Since the temperature was assumed to be the passive scalar, specification of the higher temperature at the lower wall did not cause any density-driven secondary motion. The periodic boundary condition was used in the spanwise direction assuming the flow was homogeneous in this direction. At the exit of the domain, the convective boundary condition was used in order to allow the turbulent structures to leave the domain with minimum distortion.

\subsection{Basic Flow Characteristics}

It would be instructive to remind the overall flow characteristics before the effect of external forcing is discussed. Thus, in this subsection, some of the representative mean statistics from the earlier studies [3] are provided. The mean statistics were obtained by averaging the flow variables over both time and homogeneous spanwise direction. Thus, mean variables are functions of both $x$ and $y$. The mean velocity profiles at several representative locations are given in Fig. $2 a$. As expected, the velocity profile in the region without wall blowing resembles that of the typical channel flow showing very flat shape near the wall. Due to the action of the injected fluid at the wall, the mean velocity profiles are lifted away from the wall. Similar behavior is observed for the wall-normal velocity. In the case of vertical velocity, the magnitude of the wall injection linearly increases and, thus, the location of the maximum is shifted progressively further away from the wall.

It is believed that the turbulent structures are lifted away from the wall due to the action of wall blowing and this, in turn, causes the larger turbulence production away from the wall. It is obvious that the locations of maximum streamwise and vertical velocity fluctuations are gradually moved away from the wall in Fig. 2b. Reference [3] could provide other statistics of turbulent structures including a passive scalar and the magnitude of turbulent transport terms such as Reynolds shear stress and turbulent heat flux.

Figure 3 shows that the instantaneous axial velocity profiles, $u(y)$, in the mid plane (i.e., in the plane $z / h=3.25$ ) at several streamwis locations, fluctuate significantly with time. The velocity profiles are shown only in the region close to the wall (i.e., at $y / h<0.1$ ). It was found that the nondimensional time-scale associated with this fluctuation of the velocity profile was roughly 

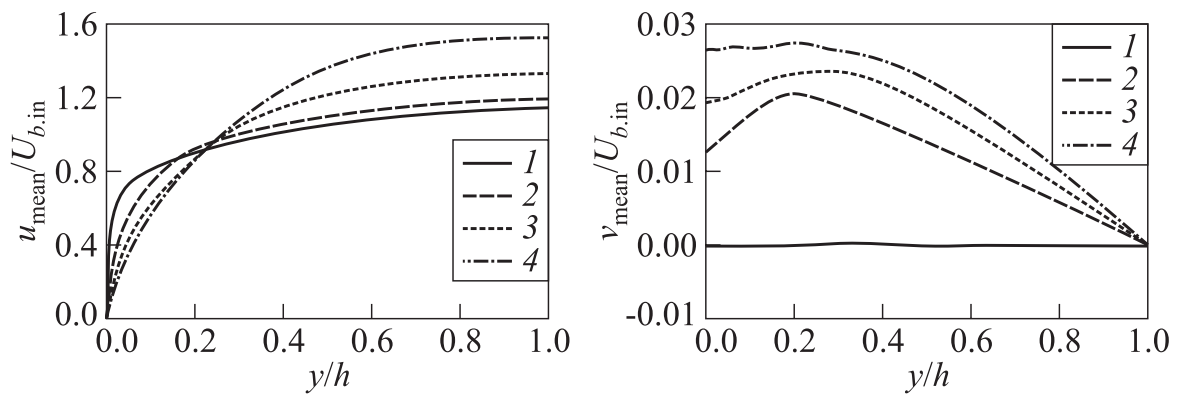

(a)
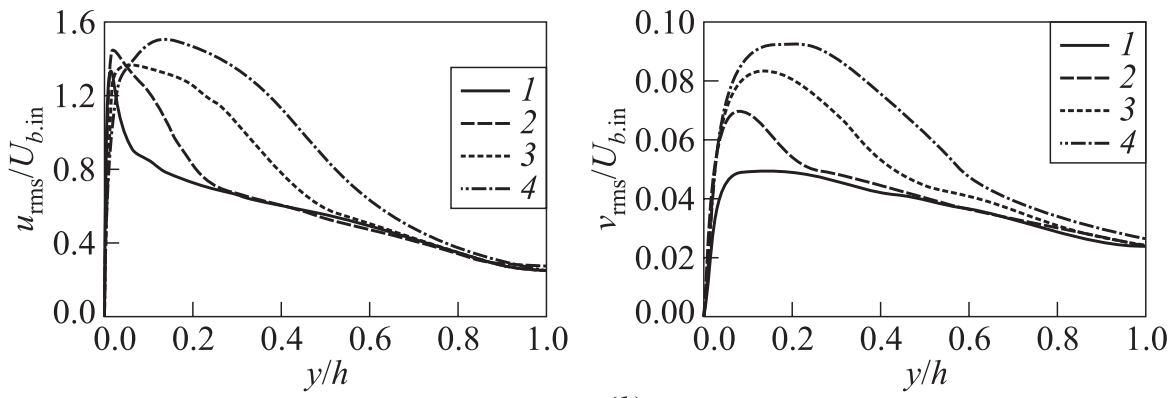

(b)

Figure 2 Mean velocity $(a)$ and rms $(b)$ profiles at several locations: $1-x / h=9.1$; $2-15.2 ; 3-19.3$; and $4-x / h=23.8$

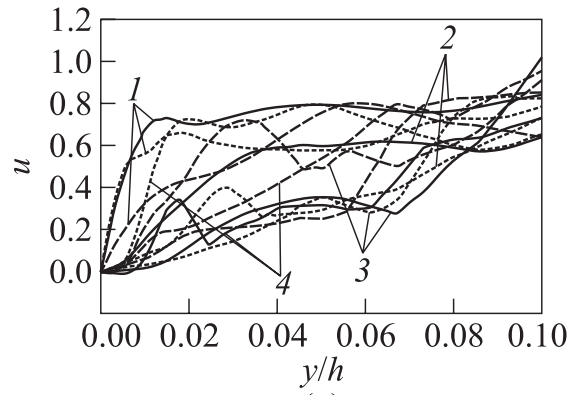

(a)

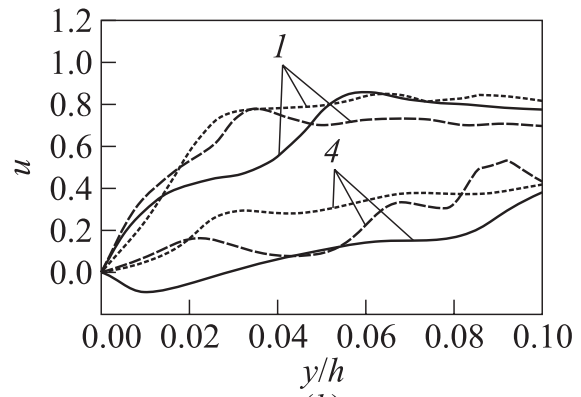

(b)

Figure 3 Instantaneous $u$ velocity profile within a cycle with blowing: $3 \%(a)$ and $5 \%(b): 1-x / h=9.1 ; 2-15.2 ; 3-19.3$; and $4-x / h=23.8$. Solid curves refer to $t=0$; dashed to $t=0.75$; and dotted to $t=1.35$ 


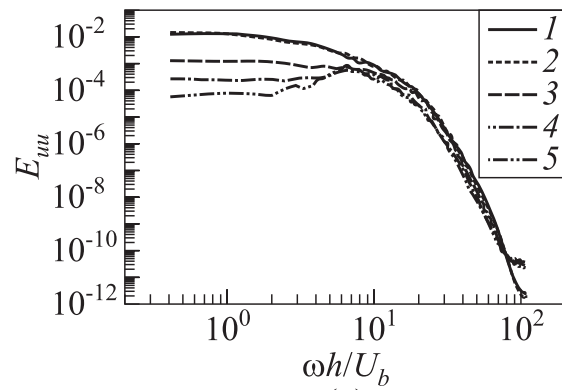

(a)

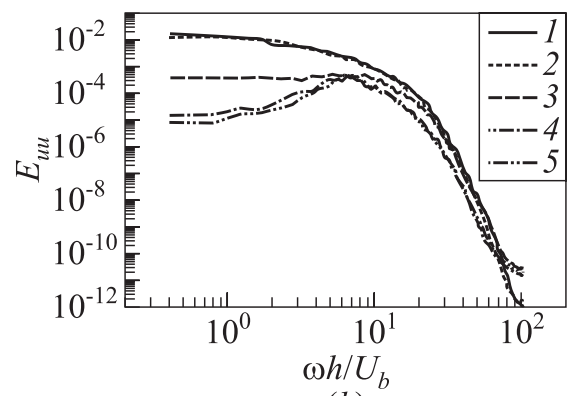

(b)

Figure 4 Frequency spectra of $u$ velocity close to the wall with blowing $3 \%(a)$ and $5 \%(b): 1-x / h=9.1 ; 2-12.1 ; 3-16.2 ; 4-20.3$; and $5-x / h=24.3$

1.35 for both cases of $3 \%$ and $5 \%$ wall injection. The velocity in the upstream location at $x / h=9.1$ exhibits a typical profile usually observed in a fully developed turbulent flow with a sharp velocity gradient near the surface. The addition of wall blowing to the main flow modifies the wall boundary layer by pushing the low-momentum fluid away from the surface and, therefore, the velocity gradient diminishes gradually to zero downstream of the flow. This behavior can be clearly seen at locations $x / h=15.2$ and 19.3. However, the velocity at $x / h=23.6$ begins to experience negative fluctuations in the region near the surface. It is believed that this negative excursion is due to the passage of roller-like vortices and is the manifestation of the periodic oscillations near the surface. The addition of wall blowing upstream seems to be strong enough to form roller-like vortices far downstream which are identified by the pressure field (will be shown later), which is thought to be responsible for the periodically oscillatory motion near the surface. The excursion of the negative velocity can be larger when the magnitude of wall blowing increases to $5 \%$ as shown in Fig. $3 b$.

One of the interesting features of the present calculations is displayed in the frequency spectra of the streamwise velocity component observed near the wall as shown in Fig. 4. At $x / h=24.3$, a peculiar local peak was found near the frequency of $\omega_{f} h / U_{b}=8.5 \sim 9$. Note that this is the streamwise location where the effect of wall blowing is quite strong. It is also interesting to note that the flow at $x / h=16.2$ does not experience oscillations even though the wall blowing is imposed. The angular frequency can be converted to the frequency value $f$ of $300 \sim 400 \mathrm{~Hz}$ if the average velocity of the liquid oxidizer is about $30 \mathrm{~m} / \mathrm{s}$ and half channel height is $0.1 \mathrm{~m}$. At this point, it is not yet clear what is the reason for a relatively larger amplification of flow oscillations near the surface in the aft section of the domain. Nevertheless, it is, at least, clear that wall blowing plays some role in rapidly modifying the near-wall turbulence structure. 


\subsection{Momentum Forcing Procedure}

The study of the response of the turbulent flow near the wall surface to external excitations is described in this subsection. Possibly, there are many different ways of imposing external forcing but momentum disturbances were chosen here because of their convenience in the context of present numerical algorithm. In order to minimize the disturbance due to the artificial forcing still providing physically realistic turbulence in the computational domain with wall blowing, the manipulation of the flow was performed in the recycled channel. In the present study, among several possible methodologies, an attempt of prescribing the external forcing with a specified frequency $\left(\omega f_{h} / U_{b}=8.5\right.$ in the current study) at the inlet was made. More specifically, the velocity increment in the form of $\sum_{n} c_{n} a_{n}(y) \cos \left(\omega_{n} t\right)$ was added to the streamwise velocity component at the inlet of the computational domain (which was basically obtained from the recycled plane). Here, $c_{n}$ is the adjustable constant which defines the strength of the intended forcing. Another constant $a_{n}(y)$ is taken to be $u_{\mathrm{rms}}(y)$ for a typical channel flow, which is introduced to prevent the situation when the external forcing unduly surpasses the recycled velocity component. Thus, the instantaneous streamwise velocity component with forcing (at $x=0$ ) had the following form:

$$
\left.u(y, z, t)\right|_{x=0}=\left.u(y, z, t)\right|_{\text {recycled plane }}+\sum_{n} c_{n} a_{n}(y) \cos \left(\omega_{n} t\right) .
$$

Having specified the velocity increment as explained above, the inlet flow, which may suffer the mild breakdown of turbulence, has a sufficient room for the redevelopment in the recycled channel.

\section{RESULTS}

The modification of near-wall turbulent structures was examined by inspecting various instantaneous as well as averaged statistics of streamwise velocity and pressure. Some of earlier works on this subject can be found elsewhere [3, 12]. For reference, it should be mentioned that wall blowing starts from the middle of the computational domain $(x / h=13.2)$ so that location $x / h=8.1$ corresponds to the place where the statistics are similar to those in a simple channel without wall injection.

\subsection{Concentrated Momentum Forcing at $\omega_{f} h / U_{b}=8.5$}

First, concentrated momentum forcing with $\omega_{f} h / U_{b}=8.5$ was imposed on the turbulent inflow with a maximum $10 \%$ magnitude of the rms value. Thus, $c_{n}$ 


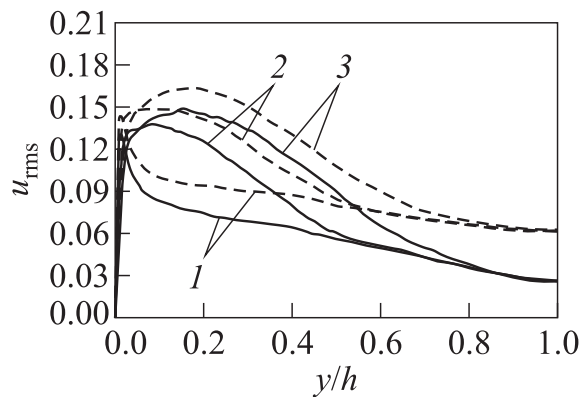

(a)

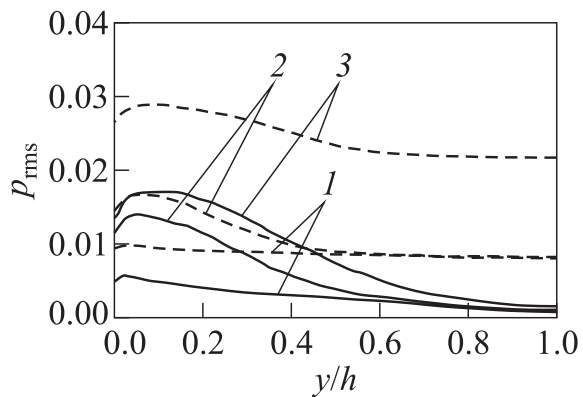

(b)

Figure 5 The rms values ((a) streamwise velocity and (b) pressure) with (dashed curves) and without (solid curves) forcing at $\omega_{f} h / U_{b}=8.5: 1-x / h=9.1 ; 2-19.2$; and $3-x / h=23.8$

in Eq. (4) was set to 0.1. The target value of $\omega_{f} h / U_{b}=8.5$ was obtained from the results of previous work [3]. As explained earlier, the interaction of mean flow with wall blowing results in this particular time-scale. Thus, forcing at this specific frequency was imposed.

Figure 5 shows the comparison of rms values of streamwise velocity and pressure with and without momentum forcing at the given frequency. This figure suggests that the overall enhancement of turbulence is due to the external excitation. The reason for the larger increase in pressure fluctuation is the assumption of incompressibility. This behavior may suggest that effective external forcing can lead to a significant amplification of the residing flow oscillations.

Time characteristics of the flow with external forcing can be analyzed in a different way by examining the time history of streamwise velocity fluctuation and pressure fluctuation components as shown in Fig. 6. This figure shows the time histories of streamwise velocity fluctuations and pressure fluctuations in the vicinity of the wall. It reveals how much the flow field near the wall is modified by the momentum forcing. Regardless of the streamwise location, the instantaneous velocity can be negative for a significant time interval indicating that the wall layer vibrates forward and backwards. Note that the relative magnitude of turbulent fluctuations is reduced in the region of wall blowing as compared to Fig. 2b. Since the effect of regression is expressed by wall blowing in the present work, it is thought that the effect of regression is to suppress the turbulent fluctuation near the wall. It is not easy to distinguish any periodic oscillatory motion with a peak frequency observed in the frequency spectra in Fig. 4.

The time history of streamwise velocity includes all components of velocity contribution such as mean value, turbulent random motion, and periodic oscillating component. However, the pressure data clearly exhibit the presence of very organized characteristics when momentum forcing is imposed. Thus, it would 


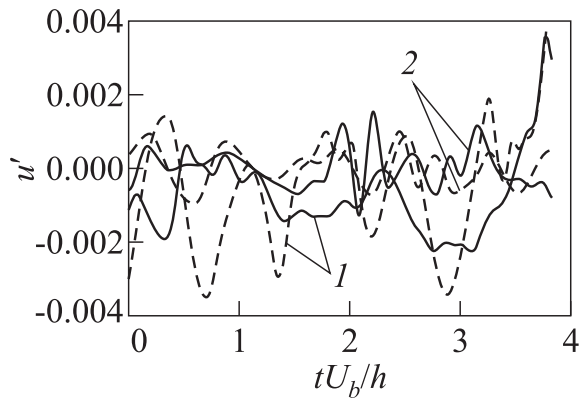

(a)

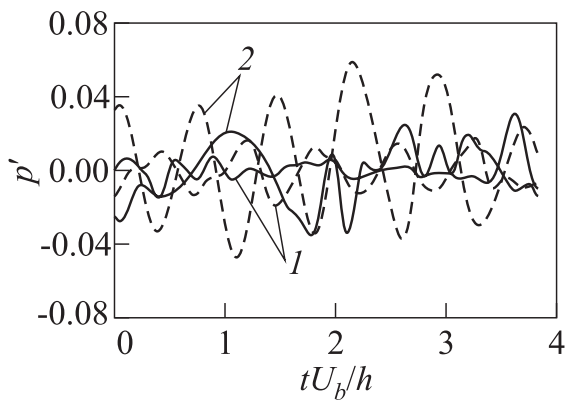

(b)

Figure 6 Time histories in the vicinity of the wall: (a) streamwise velocity fluctuations and $(b)$ pressure fluctuations with (dashed curves) and without (solid curves) forcing at $\omega_{f} h / U_{b}=8.5: 1-x / h=9.1$; and $2-x / h=23.8$

be interesting to see what nature of momentum forcing causes the occurrence of this behavior. Even though the axial velocity at various locations experiences periodic oscillations with the same frequency as shown in Fig. 4, the fluctuating component of pressure downstream of the flow is amplified very rapidly. This may be one of the evidences that vortices gain downstream more energy from the external excitation and can induce relatively large negative excursion of the instantaneous axial velocity near the surface. In order to pursue this issue, the evolution of turbulent structures should be further investigated in the near future.

To see the effect of external forcing on turbulent energy distribution in the wave-number space, the frequency spectra of streamwise velocity and pressure at two representative locations were investigated. The momentum forcing affected energy contents in the flow. Note that location $x / h=9.1$ corresponds to the region where wall blowing is absent whereas location $x / h=23.8$ represents the region where wall blowing is activated.

Figure $7 a$ shows the effect of streamwise momentum forcing at $\omega_{f} h / U_{b}=8.5$ for location $x / h=9.1$. Due to external forcing, there exists a peak at the energy spectra around frequency $\omega_{f} h / U_{b}=8.5$. One can infer how much energy is added due to momentum forcing. For both streamwise velocity and pressure, external forcing results in a sudden increase around $\omega_{f} h / U_{b}=8.5$ but it is not clear whether there is an appreciable amount of enhancement. Since the different streamwise locations have different turbulence intensities, normalization by the mean square values of fluctuation was attempted so that the integrated area under the curve was the same. To reveal the relative growth of the energy, the linear scale was used in Fig 8. Again, the increase of energy around $\omega_{f} h / U_{b}=8.5$ for location $x / h=23.8$ is obvious but it is not clear whether this increase is relatively larger than that of location $x / h=9.1$. From the results, it appears 


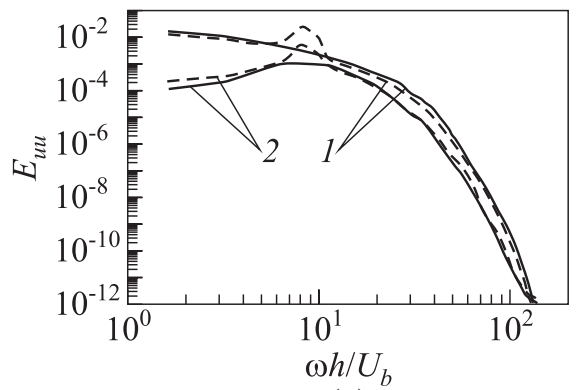

(a)

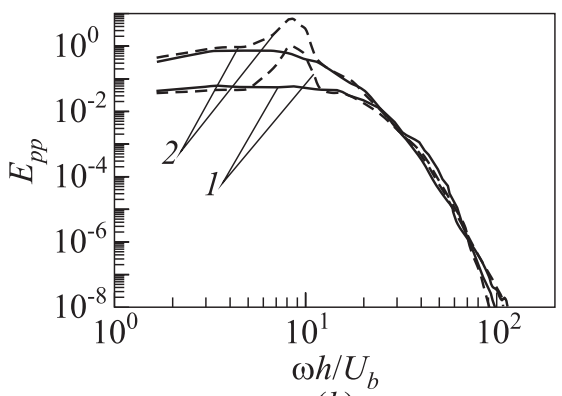

(b)

Figure 7 Frequency spectra with (dashed curves) and without (solid curves) forcing at $\omega_{f} h / U_{b}=8.5:(a)$ streamwise velocity and $(b)$ pressure at $x / h=9.1(1)$ and $x / h=23.8(2)$

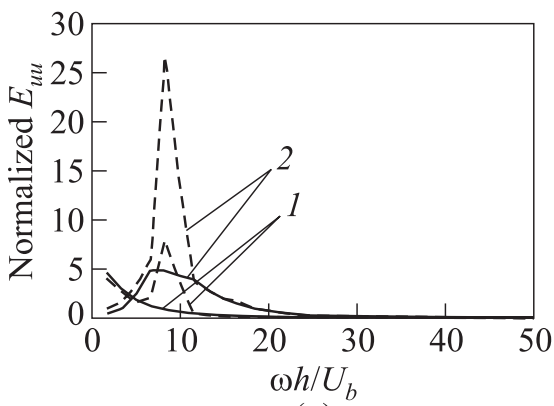

(a)

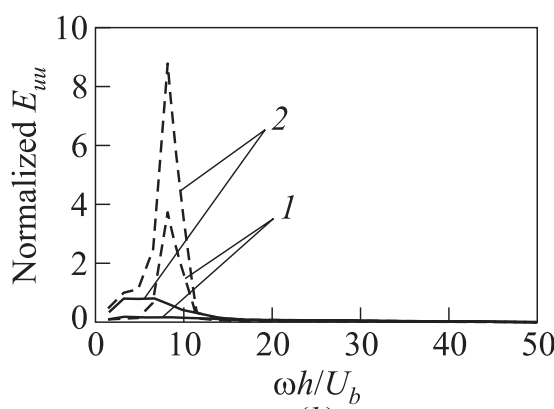

(b)

Figure 8 Normalized frequency spectra with (dashed curves) and without (solid curves) forcing at $\omega_{f} h / U_{b}=8.5:(a)$ streamwise velocity and $(b)$ pressure at $x / h$ $=9.1(1)$ and $x / h=23.8(2)$

that the amplification at location $x / h=23.8$ is the direct consequence of the input given by external forcing.

The effect of momentum forcing was studied by detecting pressure fluctuations in the $3 \mathrm{D}$ configuration. Figure 9 displays the isocontours of pressure fluctuation in the baseline case (i. e., case without forcing) whereas Fig. $9 b$ shows how the pressure field is modified by the concentrated forcing.

Figure 9 displays the roll-up process as the flow evolves downstream. In fact, this roller-like structure is not evident in the velocity or vorticity contours but isocontours of pressure fluctuations suggest the existence of Helmholtz type instability resulting from the interaction of the mean flow with wall blowing. Even though the rms values are increased due to the forcing, a stronger roll-up process is not found here. 


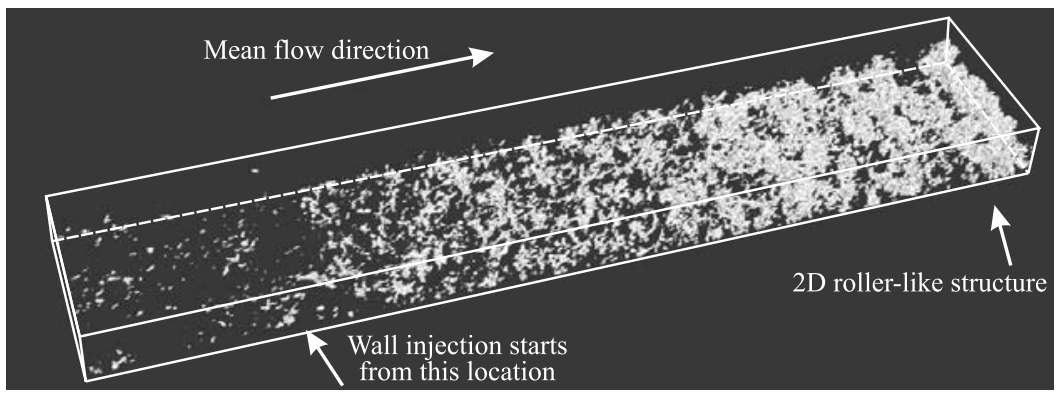

(a)

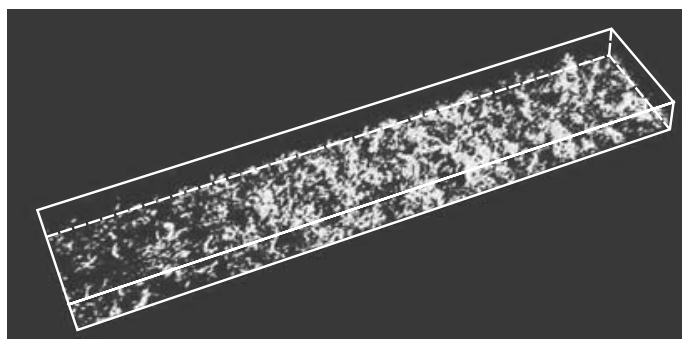

(b)

Figure 9 Isocontours of pressure fluctuation of the baseline computation $(a)$ and with forcing at $\omega_{f} h / U_{b}=8.5(b)$

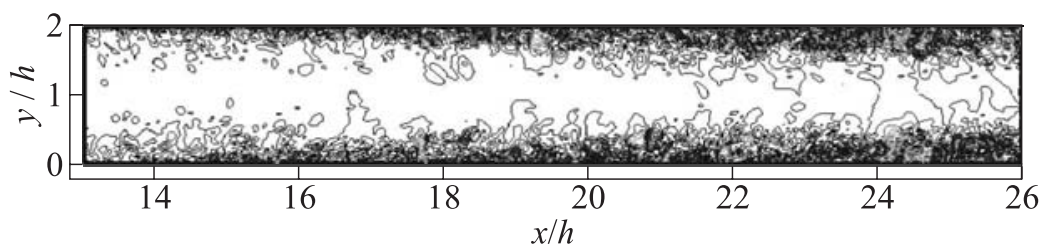

(a)

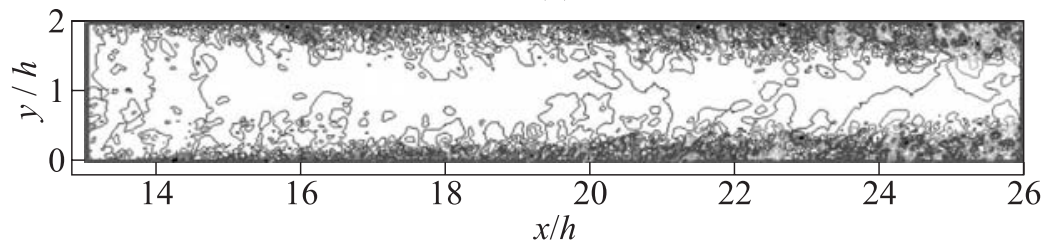

(b)

Figure 10 Contours of pressure fluctuations for baseline computation $(a)$ and with forcing at $\omega_{f} h / U_{b}=8.5(b)$ in the $x-y$ plane 
The similar behavior can be found in the $2 \mathrm{D}$ contours of pressure fluctuations detected in the middle of the computational domain $(z / h=3.25)$ in Fig. 10 . As mentioned earlier, it is obvious that the turbulence intensity increases due to momentum forcing but this increase appears to be linked to the fact that the fluctuations have a wide range of frequencies rather than strengthen the turbulence. More investigation will be necessary for better understanding of this behavior.

\subsection{Distributed Momentum Forcing at $\omega_{f} h / U_{b}=8.5 \ldots 32$}

Usually, the modification of geometry or flow configuration in the rocket motor is equivalent to imposing a specific frequency. In many operational conditions, however, white noises generated by many different means are added to the flow field. As explained earlier, the present hybrid motor exhibits a particular time scale which corresponds to $\omega_{f} h / U_{b}=8.5$. Thus, it was attempted to simulate the situation when the white noises are added to the flow field. In the present work, it was devised that the added momentum forcing had a frequency characteristics in the range of $4 \leq \omega_{f} h / U_{b} \leq 32$.

Figure 11 indicates that the effect of streamwise momentum forcing does not clearly appear in the velocity spectra. However, as found in the case of the concentrated forcing, there is a nonnegligible change in the pressure spectra. Especially, at location $x / h=23.8$, the peak near $\omega_{f} h / U_{b}=8.5$ is significantly increased. Even if the spectra are normalized again so that the area under the curve is the same, the similar behavior can be found (Fig. 12). At the moment, it appears that the effect of distributed forcing on the turbulent velocity field is negligible but it can become appreciable for the pressure field.

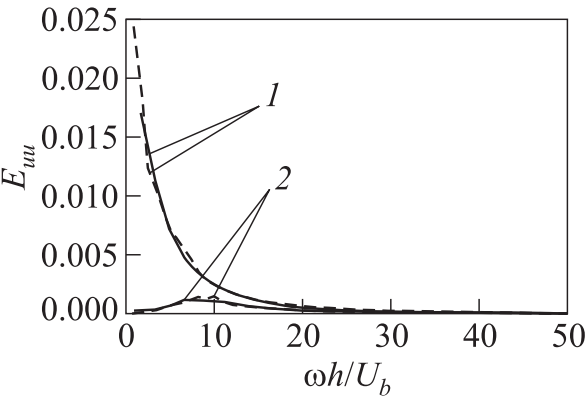

(a)

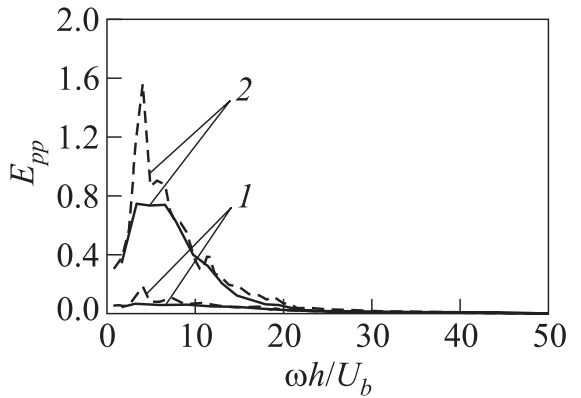

(b)

Figure 11 Frequency spectra with (dashed curves) and without (solid curves) distributed forcing: (a) streamwise velocity and $(b)$ pressure at $x / h=9.1$ (1) and $x / h=23.8(2)$ 


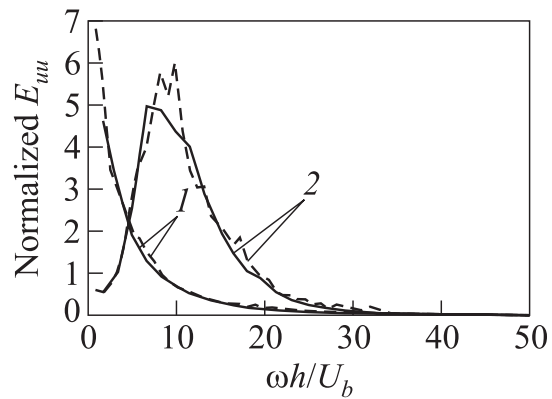

(a)

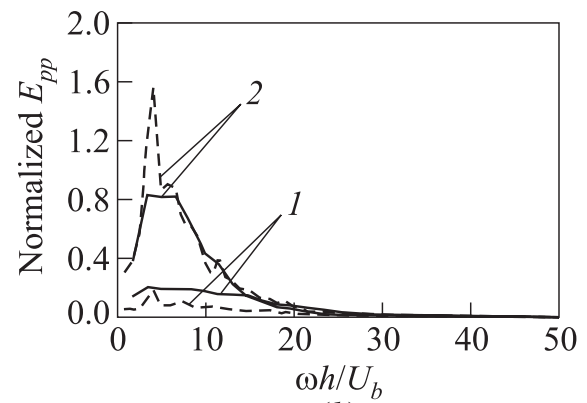

(b)

Figure 12 Normalized frequency spectra with (dashed curves) and without (solid curves) distributed forcing: (a) streamwise velocity and (b) pressure at $x / h=9.1$ (1) and $x / h=23.8(2)$

To explain the behavior shown in Figs. 11 and 12, the time-scale which corresponds to the distributed forcing should be correlated to the time scale of background turbulence and the modification of turbulent structures should be analyzed.

\section{SUMMARY}

The LES analysis has been conducted in a planar simple channel with imposed wall blowing for investigating the interaction between the main oxidizer axial flow and wall injected flow occurring due to fuel evaporation. In the present analysis, chemical reactions were not taken into account.

The calculations were conducted to see how the flow in the vicinity of the wall reacts to the external perturbations excited at the same frequency as found from the baseline simulations in the hybrid motor. The computational results showed that external momentum forcing either at a specific frequency $\left(\omega_{f} h / U_{b}=8.5\right)$ or over the specified range of frequency $\left(4 \leq \omega_{f} h / U_{b} \leq 32\right)$ can agitate the pressure a bit more than the streamwise velocity. This is believed to be due to the assumption of incompressibility where the pressure variation is about twice the relative velocity fluctuation. Since several works found in the literature on solid rocket reported that the white noise would not agitate the near-wall turbulence, the present work confirms that the distributed forcing which simulates the white noise is not very efficient for the abrupt enhancement of the pressure. However, several results imply that the pressure reacts quite sensitively and thus, this still leaves a room for possible way of building a sudden increase of flow instability. This will be the authors' future work. 


\section{REFERENCES}

1. Na, Y., and C. Lee. 2007. LES studies on the channel flow with wall bowing. AIAA Paper No. 2007-5357.

2. Na, Y., and C. Lee. 2008. Intrinsic flow oscillation in channel flow with wall blowing. AIAA Paper No. 2008-5019.

3. Lee, C., and Y. Na. 2009. Large eddy simulation of flow development in chamber with surface mass injection. J. Propul. Power 25(1).

4. Evans, B., N. A. Favorito, and K. K. Kuo. 2006. Oxidizer-type and aluminum particle addition effects on solid fuel bruning behavior. AIAA Paper No. 2006-4676.

5. Koo, W. and C. Lee. 2007. The combustion characteristics of oxidizer flow change in hybrid rocket. 2007 Korean Society of Propulsion Engineering Spring Conference Proceedings. KSPE, Seoul. 103-15.

6. Nicoud, F., T. J. Poinsot, and H.H. Minh. 1995. Direct numerical simulation of a turbulent flow with massive uniform injection. 10th Symposium on Turbulent Shear Flows Proceedings. Pennsylvania State University, U.S.A. 3:13-18.

7. Apte, S., and V. Yang. 2001. Unsteady flow evolution in porous chamber with surface mass injection. Part 1: Free oscillation. AIAA J. 39(8):1577-86.

8. Apte, S., and V. Yang. 2002. Unsteady flow evolution in porous chamber with surface mass injection. Part 2: Acoustic excitation. AIAA J. 40(2):244-53.

9. Apte, S., and V. Yang. 2003. A large-eddy simulation study of transition and flow instability in a porous-walled chamber with mass injection. J. Fluid Mech. 477:21525.

10. Na, Y. 2005. Direct numerical simulation of turbulent scalar field in a channel with wall injection. Numer. Heat Transfer A 47:165-81.

11. Lund, T., X. Wu, and K. D. Squires. 1998. Generattion of turbulent inflow data for spatially-developing boundary layer simulation. J. Comput. Phys. 140:233-58.

12. Rho, T.S., and T.E. C. Culick. 1998. Numerical study of transient combustion responses to acoustic oscillations in axisymmetric rocket motors. AIAA Paper No. 980253 . 